University for Business and Technology in Kosovo

UBT Knowledge Center

UBT International Conference

2016 UBT International Conference

Oct 28th, 9:00 AM - Oct 30th, 5:00 PM

\title{
Some Propositions about Inverse Semigroups
}

Osman Hysa

Aleksander Moisiu University, o.hysa@yahoo.com

Arben Reka

Aleksander Moisiu University

Follow this and additional works at: https://knowledgecenter.ubt-uni.net/conference

Part of the Social and Behavioral Sciences Commons

\section{Recommended Citation}

Hysa, Osman and Reka, Arben, "Some Propositions about Inverse Semigroups" (2016). UBT International Conference. 29.

https://knowledgecenter.ubt-uni.net/conference/2016/all-events/29

This Event is brought to you for free and open access by the Publication and Journals at UBT Knowledge Center. It has been accepted for inclusion in UBT International Conference by an authorized administrator of UBT Knowledge Center. For more information, please contact knowledge.center@ubt-uni.net. 


\title{
SOME PROPOSITIONS ABOUT INVERSE SEMIGROUPS
}

\author{
Osman Hysa ${ }^{1}$, Arben Reka ${ }^{2}$ \\ Head of the Mathematics Department at the "Aleksandër Moisiu“ University, Durrës, \\ Albania \\ Mathematics Department of the “Aleksandër Moisiu“ University, Durrës, Albania
}

\begin{abstract}
The inverse semigroups are semigroups studied by many algebraists. In this paper we will formulate and prove some other propositions on these semigroups. So we will prove two propositions concerning the closure of a subsemigroup of a given inverse semigroup $\mathrm{S}$, within the meaning introduced by Schein in 1962, two propositions on the group congruence on a normal subsemigroup of the inverse semigroup $\mathrm{S}$, and a proposition about closed subsemigroup assertion of an inverse semigroup $\mathrm{S}$.
\end{abstract}

Keywords: closure of the semigroup, group congruence, closed semigroup

\section{Some definitons}

Definition 1. [1] A subsemigroup of the given semigroup $S$ is called full subsemigroup if it contains all the idempotents of this semigroup $S$.

In 1952, Vegner [5] introduced a natural partial order on an inverse semigroup $S$ as follows:

$$
a \leq b \Leftrightarrow \exists e \in E(S), a=e b
$$

If $H$ is an arbitrary subset of an inverse semigroup $S$, then, Schein in [3] and Clifford and Preston in [4], give this:

Definition 2. The closure of $H$ is the set $H \omega$ defined as below:

$$
H \omega=\{x \in S \mid \exists h \in H, h \leq x\}
$$

From this definition we see, immediately, that if $H$ and $K$ are subsets of $S$ than:

$$
H \subset H \omega ; H \subset K \Rightarrow H \omega \subset K \omega \text { and }(H \omega) \omega=H \omega
$$

Definition 3. [1], [2] The subset $H$ of the inverse semigroup $S$ is called closed if we have $H \omega=H$ Definition 4. [2] The subsemigroup $N$ of the inverse semigroup $S$ will be called normal if it is full, closed and $\forall x \in S, \quad x^{\prime} N x \subset N$ where $x$ ' is an inverse element of $x$.

Definition 5. [2], [6], [7], [8] A congruence $\rho$ on an inverse semigroup $S$ will be called a group congruence if the factor semigroup $S / \rho$ is a group.

Now, we must prove this propositions:

Proposition 1. If $S$ is an inverse semigroup and $E$ is the set of its idempotents then E $\omega$ is an Proof. First we see that: normal inverse subsemigroup of $S$. 
Book of Proceedings

International Conference on Social Sciences, Humanities, and other sciences

$$
\begin{aligned}
& (x \in E \omega) \Rightarrow(\exists e \in E, e \leq x) \Rightarrow\left(\exists e_{1} \in E, e=e_{1} x\right) \Rightarrow\left(e_{1} x x^{\prime}=e x^{\prime}\right) \Rightarrow \\
& \left(e_{1} e_{2}=e x^{\prime}\right) \Rightarrow\left(e^{\prime}=e x^{\prime}\right) \Rightarrow\left(e^{\prime} \leq x^{\prime}\right) \Rightarrow\left(x^{\prime} \in E \omega\right)
\end{aligned}
$$

where $e_{1}, e_{2}, e^{\prime} \in E, e_{2}=x x^{\prime}, e_{1} e_{2}=e^{\prime}$ and $x^{\prime}$ is an inverse element of $x$. So, we have shown that $(x \in E \omega) \Rightarrow\left(x^{\prime} \in E \omega\right)$, i.e $E \omega$ is inversive subsemigroup of $S$. Second, $E \omega$ is also full inverse subsemigroup of $S$, because $E \subset E \omega$, that means $E \omega$ contains all idempotents of $S$.

Third,

$E \omega$ is closed subsemigroup of $S$, because $(E \omega) \omega=E \omega$.

Finally,

we must shown that $\forall x \in S, x^{\prime} E \omega x \subset E \omega$. Indeed we have:

$$
\left(y \in x^{\prime} E \omega x\right) \Rightarrow\left(\exists z \in E \omega, y=x^{\prime} z x\right) \quad \text { and }
$$

$$
\begin{aligned}
& (z \in E \omega) \Rightarrow(\exists e \in E, e \leq z) \Rightarrow(\exists f \in E, e=f z) \Rightarrow\left[\left(x x^{\prime}\right) e=\left(x x^{\prime}\right)(f z)\right] \\
& \Rightarrow\left[\left(x x^{\prime}\right) e=f\left(x x^{\prime}\right) z\right] \Rightarrow\left[\left(x x^{\prime}\right) e=(f x)\left(x^{\prime} z\right)\right] \Rightarrow\left[\left(x x^{\prime}\right) e x=(f x)\left(x^{\prime} z x\right)\right] \\
& \Rightarrow\left[e\left(x x^{\prime}\right) x=(f x) y\right] \Rightarrow[e x=(f x) y] \Rightarrow\left[x^{\prime} e x=\left(x^{\prime} f x\right) y\right]
\end{aligned}
$$

moreover, the elements $x^{\prime} e x=e^{\prime}, x^{\prime} f x=f^{\prime}$ are idempotents (Indeed $\left(x^{\prime} e x\right)^{2}=x^{\prime} e x x^{\prime} e x=$

Now, from the last equlity (2) we have $e^{\prime}=f^{\prime} y$ and $\left(e^{\prime}, f^{\prime} \in E\right) \Rightarrow\left(e^{\prime} \leq y\right) \Rightarrow(y \in E \omega) . \quad$ So, we have proved that $E \omega$ is normal inversive subsemigroup of the semigroup $S$.

Proposition 2. If $N$ is an inverse normal subsemigroup of the inverse semigroup of $S$, then we have: $\forall x \in S,(N x) \omega=(x N) \omega$

Proof. Let be $y \in(N x) \omega$ then we see that:

$$
[y \in(N x) \omega] \Rightarrow(\exists n x \in N x, n x \leq y) \Rightarrow(\exists e \in E, n x=e y) \Rightarrow\left(x^{\prime} n x=x^{\prime} e y\right)
$$

where $x^{\prime}$ is an inverse element of $x$. But $N$ is normal, that means $x^{\prime} N x \subset N$, so $x^{\prime} n x=n_{1}$ and $n_{1} \in N$. From the last equality of (1) we will have:

$$
\left(n_{1}=x^{\prime} e y\right) \Rightarrow\left(x n_{1}=\left(x x^{\prime}\right) e y\right) \Rightarrow\left(n_{1}=e^{\prime} y\right)
$$

where $e^{\prime}=\left(x x^{\prime}\right) e \in E$ as a product of two idempotents of $E$. Now we can write:

$$
\left(x n_{1}=e^{\prime} y\right) \Rightarrow\left(x n_{1} \leq y\right) \Rightarrow[y \in(x N) \omega]
$$

Finally, we have prove that $\forall x \in S,(N x) \omega \subset(x N) \omega$. It's the same to prove also $\forall x \in S,(N x) \omega \supset(x N) \omega$, so $\forall x \in S,(N x) \omega=(x N) \omega$ 
Proposition 3. If $N$ is an inverse normal subsemigroup of the inverse semigroup of $S$, then $\rho_{N}=\left\{(x, y) \in S^{2} \mid x y^{\prime} \in N\right\}$ is a group congruence on $S$, and the $\rho_{N}$ - class $N$ is the identity of the factor group $S / \rho_{N}$.

Proof. First, we must show that the relation $\rho_{N}$ is an equivalence relation on $S$.

$\forall x \in S, x x^{\prime}$ is an idempotent, so $x x^{\prime} \in E$, where $E$ is the set of idempotents of $S$. Since $N$ is normal, then it will be full. Thus $E \subset N$ that means $x x^{\prime} \in N$ or we have $x \rho_{N} x$. We have shown that $\rho_{N}$ is reflective relation.

b) $\forall(x, y) \in S^{2}$, we see:

$$
\left(x \rho_{N} y\right) \Rightarrow\left(x y^{\prime} \in N\right) \Rightarrow\left[\left(x y^{\prime}\right)^{\prime} \in N\right] \Rightarrow\left(y x^{\prime} \in N\right) \Rightarrow\left(y \rho_{N} x\right)
$$

i.e. $\rho_{N}$ is symetric relation.

c) $\forall(x, y) \in S^{2}$, we have also:

$\left(x \rho_{N} y \wedge y \rho_{N} z\right) \Rightarrow\left(x y^{\prime} \in N \wedge y z^{\prime} \in N\right) \Rightarrow\left[\left(x y^{\prime}\right)\left(y z^{\prime}\right) \in N\right] \Rightarrow\left[\left(x x^{\prime} x y^{\prime}\right)\left(y z^{\prime}\right) \in N\right] \Rightarrow$ $\left.\left.\left[x\left(x^{\prime} x\right)\left(y^{\prime} y\right) z^{\prime}\right) \in N\right] \Rightarrow\left[x\left(y^{\prime} y\right)\left(x^{\prime} x\right) z^{\prime}\right) \in N\right] \Rightarrow\left[\left(x y^{\prime}\right)\left(y x^{\prime}\right)\left(x z^{\prime}\right) \in N\right] \Rightarrow$ $\left[\left(x y^{\prime}\right)\left(x y^{\prime}\right)^{\prime}\left(x z^{\prime}\right) \in N\right] \Rightarrow\left[\left(a a^{\prime}\right)\left(x z^{\prime}\right) \in N\right]$ where $a=x y^{\prime}$ and $a a^{\prime}=e$ is an idempotent in $S$, such that $e\left(x z^{\prime}\right) \in N$. Now, if $e\left(x z^{\prime}\right)=n$, than $n \leq x z^{\prime}$ that means $x z^{\prime} \in N \omega$ or $x z^{\prime} \in N \Rightarrow x \rho_{N} z$ or $\rho_{N}$ is a transitive relation, $(N$ is normal i.e. $N$ is closed, so $N \omega=N)$. Thus we have prove that $\rho_{N}$ is an equivalence relation.

Second, we must show that $\rho_{N}$ is a congruence. Let we have $x \rho_{N} y$ and let show now that $\forall c \in S,(x c) \rho_{N}(y c)$ or $(x c)(y c)^{\prime} \in N$. Indeed,

$(x c)(y c)^{\prime}=(x c)\left(c^{\prime} y^{\prime}\right)=(x c)\left(c^{\prime} y^{\prime}\right)=\left(x x^{\prime} x\right)\left(c c^{\prime}\right) y^{\prime}=x\left(x^{\prime} x\right)\left(c c^{\prime}\right) y^{\prime}=x\left(c c^{\prime}\right)\left(x^{\prime} x\right) y^{\prime}=$ $(x c)\left(c^{\prime} x^{\prime}\right)\left(x y^{\prime}\right)=(x c)(c x)^{\prime}\left(x y^{\prime}\right)=\left(a a^{\prime}\right)\left(x y^{\prime}\right)=e\left(x y^{\prime}\right)$

where $a=x c$, and $e=a a^{\prime}$ is an idempotent of $S$. It follows that $e \in N$, because $N$ is full, that means $E \subset N$. Now we can write:

$$
\left(e \in N \wedge x y^{\prime} \in N\right) \Rightarrow\left[e\left(x y^{\prime}\right) \in N\right] \Rightarrow\left[(x c)(y c)^{\prime} \in N\right] \Rightarrow(x c) \rho_{N}(y c)
$$

so $\rho_{N}$ is a right congruence. We can see also that $x \rho_{N} y \Rightarrow x y^{\prime} \in N$ and we will need to show $(c x) \rho_{N}(c y)$. Indeed,

$$
(c x)(c y)^{\prime}=(c x)\left(y^{\prime} c^{\prime}\right)=c\left(x y^{\prime}\right) c^{\prime}=\left(c^{\prime}\right)^{\prime}\left(x y^{\prime}\right) c^{\prime}=a^{\prime}\left(x y^{\prime}\right) a \in a^{\prime} N a \subset N
$$


because $N$ is normal. Thus, $(c x)(c y)^{\prime} \in N$, that means $(c x) \rho_{N}(c y)$ and so we conclude that $\rho_{N}$ is also left congruence, i.e. congruence on $S$.

Third, we must to show that $N$ is a $\rho_{N}$ - class:

a) $\forall(x, y) \in N^{2},\left(x \in N \wedge y^{\prime} \in N\right) \Rightarrow\left(\mathrm{xy}^{\prime} \in \mathrm{N}\right) \Rightarrow x \rho_{N} y$ so, all the elements of $N$ belongs in the same $\rho_{N}$ - class.

b) $\forall n \in N, \forall x \in S, n \rho_{N} x \Rightarrow n x^{\prime} \in N$. If $n x^{\prime}=n_{1}$ we have $\left(n_{1}=n x^{\prime}\right) \Rightarrow\left[n_{2}=n^{\prime} n_{1}=\left(n^{\prime} n\right) x^{\prime}=e x\right]$ where $n_{2}=n^{\prime} n_{1} \in N$ and $n^{\prime} n=e \in E \subset N$, so we will have: $\left(n_{2}=e x^{\prime}\right) \Rightarrow\left(n_{2} \leq x^{\prime}\right) \Rightarrow\left(x^{\prime} \in N \omega\right) \Rightarrow\left(x^{\prime} \in N\right) \Rightarrow(x \in N)$. Now, from a) and b), we can conclude that $N$ is a $\rho_{N}$ - class.

Since $E \subset N$, i.e. all the idempotents belong in the same $\rho_{N}$ - class, $N$, it follows that $\rho_{N}$ is a group congruence and is clear that $N$ is the unite element of the group $S / \rho_{N}$. Indeed, fo any class $\bar{x} \in S / \rho_{N}$ we have we have $\bar{x} N=\bar{x} \cdot \bar{e}=\overline{x e}=\bar{x} \quad$ because we have $x e \rho_{N} x$ and $x e x^{\prime} \in E$ Indeed, $\left(x e x^{\prime}\right)^{2}=x e\left(x^{\prime} x\right) e x^{\prime}=x\left(x^{\prime} x\right) e e x^{\prime}=x e x^{\prime}$, so $x e x^{\prime} \in E \subset N$ that means $x e \rho_{N} x^{\prime}$ or $\overline{x e}=\bar{x}$. It is the same to show $N \bar{x}=\bar{x}$.

Proposition 4. If $\tau$ is a group congruence on $S$, than exists an inverse normal subsemigroup $N$ of $S$ such that $\tau=\rho_{N}$.

Proof. Since $\tau$ is a group congruence on $S$, it follows that $S / \tau$ is a group. Let be $N$ the $\tau$ - class that is the identity element of the group $S / \tau$. Now we will prove that $\tau=\rho_{N}$.

First, $N$ is a subsemigroup of $S$ because:

$(x \in N \wedge y \in N) \Rightarrow(\bar{x}=N \wedge \bar{y}=N) \Rightarrow\left(\bar{x} \cdot \bar{y}=N^{2}=N\right) \Rightarrow(\overline{x y}=N) \Rightarrow(x y \in N)$

Second, $N$ is the identity element of the group $S / \rho_{N}$ that means all the idempotents of $S$ belongs to $N$, i.e. $N$ is full, so $\forall x \in N, x x^{\prime}=e \in E \subset N$. On the other hand, we have:

$$
\left(x x^{\prime}=e \in N\right) \Rightarrow\left(\overline{x x^{\prime}}=\bar{e}=N\right) \Rightarrow\left(\bar{x} \cdot \overline{x^{\prime}}=N\right) \Rightarrow\left(N \overline{x^{\prime}}=N\right) \Rightarrow\left(\overline{x^{\prime}}=N\right) \Rightarrow\left(x^{\prime} \in N\right)
$$

Where $\bar{x}=N$, because $x \in N$. Thus $N$ is inversive subsemigroup of $S$.

Third, $N$ is closed subsemigroup os $S$. Indeed, 


$$
\begin{aligned}
& (x \in N \omega) \Rightarrow(\exists n \in N, n \leq x) \Rightarrow(\exists e \in E \subset N, n=e x) \\
& \Rightarrow(N=\bar{n}=\overline{e x}=\bar{e} \cdot \bar{x}=N \bar{x}=\bar{x})
\end{aligned}
$$

So, $\bar{x}=N$ that means $x \in N$ and $N \omega=N$ (the other inclusion $N \omega \supset N$ is evident from the definition of closure of $N$ ), i.e. $N$ is closed.

Fourth, we need to show that $\forall x \in S, x^{\prime} N x \subset N$. Indeed,

$$
\begin{aligned}
& \left(y \in x^{\prime} N x\right) \Rightarrow\left(y=x^{\prime} n x, n \in N\right) \Rightarrow\left(\bar{y}=\overline{x^{\prime} n x}\right) \Rightarrow \\
& \left(\bar{y}=\overline{x^{\prime}} \cdot \bar{n} \cdot \bar{x}=\overline{x^{\prime}} \cdot N \cdot \bar{x}\right) \Rightarrow\left(\bar{y}=\overline{x^{\prime}} \cdot \bar{x}\right) \Rightarrow\left(\bar{y}=\overline{x^{\prime} x}=\bar{e}=N\right)
\end{aligned}
$$

So, $\bar{y}=N$ that means $y \in N$, i.e. $N$ is normal and identity element for both grupet $S / \tau$ and $S / \rho_{N}$

Fifth, it remains to be shown that $\tau=\rho_{N}$, where $\rho_{N}=\left\{(x, y) \in S^{2} \mid x y^{\prime} \in N\right\}$, since $\tau$ is congruence, we have:

$$
(x \tau y) \Rightarrow\left[\left(x y^{\prime}\right) \tau\left(y y^{\prime}\right)\right] \Rightarrow\left[\left(x y^{\prime}\right) \tau e\right] \Rightarrow\left[{\overline{\left(x y^{\prime}\right.}}^{\tau}=\bar{e}^{\tau}=N\right] \Rightarrow\left(x y^{\prime} \in N\right) \Rightarrow\left(x \rho_{N} y\right)
$$

Thus, we have prove that $\tau \subset \rho_{N}$ (2), vice versa we have:

$$
\begin{aligned}
& \left(x \rho_{N} y\right) \Rightarrow\left(x y^{\prime} \in N\right) \Rightarrow\left(\overline{x y}^{\rho_{N}}=N\right) \Rightarrow\left(\overline{x y}^{\rho_{N}}=\overline{y y}^{\rho_{N}}=\bar{e}^{\rho_{N}}=N\right) \Rightarrow \\
& {\left[\left(x y^{\prime} \in N\right) \wedge\left(y y^{\prime} \in N\right)\right] \Rightarrow\left[\left(x y^{\prime}\right) \tau\left(y y^{\prime}\right)\right] \Rightarrow\left[\left(x y^{\prime} y\right) \tau\left(y y^{\prime} y\right)\right] \Rightarrow} \\
& {\left[\left(x e_{1}\right) \tau y\right] \Rightarrow\left(\overline{x e}_{1}^{\tau}=\bar{y}^{\tau}\right) \Rightarrow\left(\bar{x}^{\tau} N=\bar{y}^{\tau}\right) \Rightarrow\left(\bar{x}^{\tau}=\bar{y}^{\tau}\right) \Rightarrow(x \tau y)}
\end{aligned}
$$

So, it follows that we have also $\rho_{N} \subset \tau$

Now, from (2) and (3) we can conclude that $\tau=\rho_{N}$

Proposition 5. If $U$ is a closed inverse subsemigroup of $T$ where $T$ is a closed inverse subsemigroup of the semigroup $S$, then $U$ is a closed inverse subsemigroup of $S$

Proof. Let be $U \omega_{T}$ the closure of $U$ with respect to $T, U \omega_{S}$ the closure of $U$ with respect to $S$ and $T \omega_{S}$ the closure of $T$ with respect to $S$. So, we have: $U \omega_{T}=U$ and $T \omega_{S}=T$ Now, we must show that $U \omega_{S}=U$

First, we see that $U \omega_{T} \subset U \omega_{S}$, because,

$\left(x \in U \omega_{T}\right) \Rightarrow[\exists u \in U,(u \leq x) \wedge(x \in T)] \Rightarrow[\exists u \in U,(u \leq x) \wedge(x \in S)] \Rightarrow\left(u \in U \omega_{S}\right)$ 
Book of Proceedings

International Conference on Social Sciences, Humanities, and other sciences

Second, we $\quad$ see: $\quad\left(x \in U \omega_{S}\right) \Rightarrow\left(x \in T \omega_{S}\right) \quad$ because:

$(U \subset T) \Rightarrow\left(U \omega_{S} \subset T \omega_{S}=T\right)$, i.e. $x \in T$ further $U \omega_{S} \subset T \omega_{S}$, and now we can write

$\left(x \in U \omega_{S}\right) \Rightarrow\left(x \in T \omega_{S}\right) \Rightarrow[\exists u \in U,(u \leq x) \wedge(x \in T)] \Rightarrow\left(x \in U \omega_{T}=U\right) \Rightarrow(x \in U)$

Finally, we have proved that $U \omega_{S}=U$, so, $U$ is a closed inverse subsemigroup of $S$.

\section{References}

1. Roman S.Gigon."Congruences and group congruences on a semigroup”, August 2012, semigroup forum.

2. J.M.Howie, “An introduction to semigroup theory”, 1976, Academic Press, London, New York, San Francisco.

3. Bernd Billhardt (communicated by Boris Schein), "On inverse semigroups the closure of whose set of idempotents is a clifford semigroup", Semigroup forum, Vol.44 (1962), 320-331

4. Clifford a.H; Preston G,B. (1967), "The algebraic theory of semigroups", Vol. II.

5. Vegner (1952), “Generalized groups”, Dokl.Akad. Nauk SSSR, Vol. 84, 1119-1122, (Russian)

6. J.M. Howie, Proc. Edinb. Math. Soc. 14, 71-79, 19-64

7. Hanumantha Rao, S. Lakshmi P. "Group congruences on eventually regular semigroups", J. Aust. Math. Soc. 45A, 320-325 (1988)

8. La Torre, D. R.: “Group congruences on regular semigroups”, Semigroup forum 30, 1-16, 1984 